\title{
Leakage Current in P-N Junction Diode by Influent from SRFE Process
}

\author{
Narong Sangwaranatee, Itsara Srithanachai, Surasuk Niemcharoen
}

\begin{abstract}
This paper present the effect from soft radiation flash exposure (SRFE) on electrical properties of semiconductor device. SRFE process take only few second on semiconductor device but it quite impact to device performance. The part we will study impact from SRFE such as surface and bulk. COMSOL is tool for help to understand more detail in term of surface recombination, temperature and bulk effect. Surface of device get impact from radiation and temperature generate from radiation. Also, silicon bulk get damage from radiation due to has high penetrate. From electrical results show that leakage current of device has reduce after SRFE process, mean radiation can help to improve or recover damage from fabrication process.
\end{abstract}

Keywords: SRFE, Leakage current, P-N junction diode, Defect.

\section{INTRODUCTION}

Semiconductor material is important for electronics device because can be modify characteristics by application requirement such as switching diode has to lightly P-region doped and heavy N-regions doped [1] and transistor (BJT) constructed with 3 doped semiconductor regions [2]. Semiconductor material can use for many applications and always improve performance around $40 \%$ every $1.5-2$ years by refer from "Moore law" [3].

Many years ago, we use semiconductor material in satellite application and sent to space [4]. In space consist of many kind of radiation and impact to device performance and lifetime because radiation has high penetrate, high energy, no mass and no electrical charge. The application use semiconductor material such as solar cell for generate energy [5], detector for measure radiation [6] and temperature [7].

Radiation in the space has separate by wavelength and frequency for example gamma [8, 9], neutron, proton [10] and roentgen [11]. One of radiation still using in many application is X-ray or Roentgen. Although, Roentgen always use but also

Revised Manuscript Received on April 25, 2020.

* Correspondence Author

Narong Sangnarawatee*, Applied Physics Research Group, Faculty of Science and Technology, Suan Sunandha Rajabhat University, Bangkok 10300, Thailand. Email: narong.sa@ssru.ac.th

Itsara Srithanachai, Department of Electronics, Faculty of $\mathrm{K}$ ngineering,King Mongkut's Institute of Technology Ladkrabang, Bangkok 10520, Thailand. Email: srithanachai@gmail.com

Surasak Niemcharoen, Department of Electronics, Faculty of $\mathrm{K}$ ngineering,King Mongkut's Institute of Technology Ladkrabang, Bangkok 10520, Thailand. Email: knsurasa@kmitl.ac.th

(C) The Authors. Published by Blue Eyes Intelligence Engineering and Sciences Publication (BEIESP). This is an open access article under the CC BY-NC-ND license (http://creativecommons.org/licenses/by-nc-nd/4.0/) risk for user if get high dose. However, Roentgen has research for several year of the characteristics because we try to apply by various frequency and energy in many application.

COMSOL is one of software for simulation semiconductor characteristics before fabrication. Software allow put multi-factors and processing close to real effect with fabrication device. The flexible of program can adjust parameters and review influent of some part, also can save cost and time for research company.

The goal of this paper study impact of SRFE process on semiconductor device by analysis electrical characteristics and also using simulation program confirm effect from radiation on surface and substrate bulk. The effect we are study in this paper such as doping range, temperature on device and concentration in doped region.

\section{EXPERIMENT PROCEDURE}

$\mathrm{P}-\mathrm{N}$ diode of this paper fabricated under CMOS theory and technology at Thai Microelectronics Center (TMEC). Device design by using COMSOL program before fabricate. The fabrication process flow by: (a) dry etching after deposited oxide cover substrate, (b) doped phosphorus in active area by implantation with $120 \mathrm{eV}$ on backside of substrate, (c) doping boron on front side by same energy of backside, (d) annealing by RTA process for few minute and (e) growth $\mathrm{Al}$ around 10 um thick both side. The device after fabricate show in Fig. 1. [12]

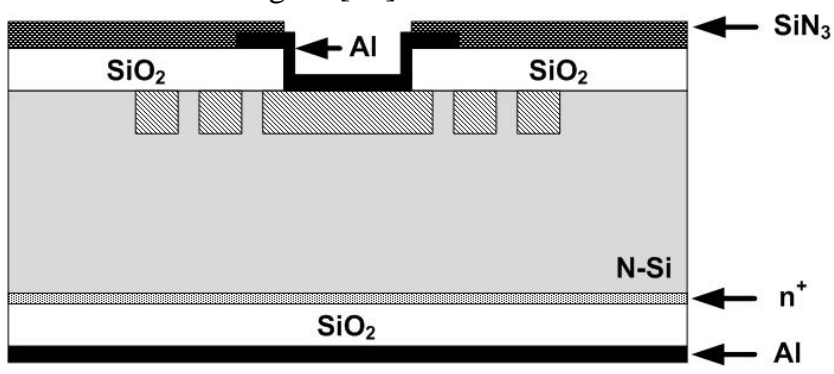

Fig.1 Device structure

Device will measure electrical properties by using semiconductor analyzer device model B1500A. I-V characteristics of device will bias by sweep voltage from -10 to $1 \mathrm{~V}$ with frequency $100 \mathrm{kHz}$ in room temperature. After measure electrical properties, device will exposed by Roentgen for few second for many time to reach target dose. SRFE process show in Fig. 2. [13]

Simulation results using COMSOL semiconductors mode for study impact from radiation on surface and substrate bulk. We input many parameter in program such as doping concentration, radiation energy, time of exposure and bias voltage.

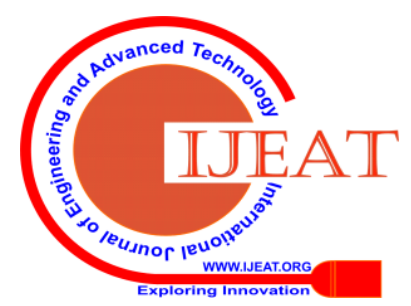




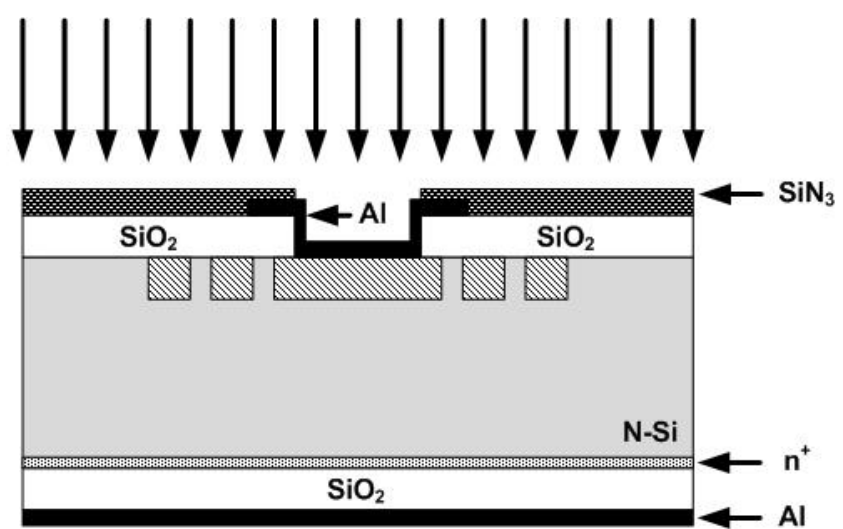

Fig. 2 SRFE process on device

\section{RESUTLS AND DISCUSSIONS}

From Fig. 3 shows simulation results the concentration of boron doped and deep level after annealing process (RTA). In principle, doping atom after implantation will deep in to substrate bulk around 1-2 $\mu \mathrm{m}$ but after RTA will deep to around 6-7 $\mu \mathrm{m}$ depend on temperature, time, atom mobility and doping concentration. From the results Fig.3a the concentration in active area surface show lower concentration due to atom deep into silicon bulk. Moreover, doping atom diffuse to guard ring. Atom will deep to bulk from surface shown in Fig. 3b then concentration decrease when over $7 \mu \mathrm{m}$ show in yellow, green color in Fig. 3c.

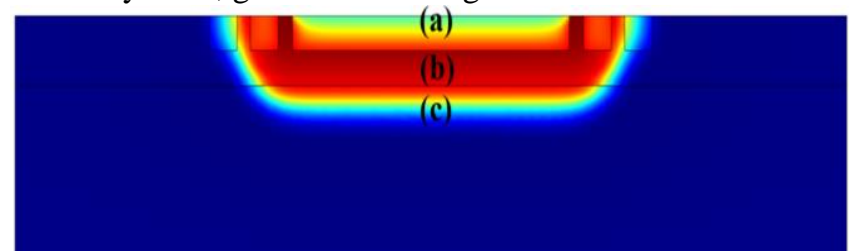

Fig. 3. Diffusion range of boron atom doped after RTA process

The concentration range show in Fig. 4. Results can explain more detail in Fig.3, the concentration of doping atom reduced when deep into substrate bulk.

From both Fig.3 and Fig. 4 can confirm evidence from boron atom doped in substrate bulk for few micrometers and make device effective in term of P-N junction. While boron doped to silicon structure it will be happen for host mechanism. This effect can generate defect in device structure such as generate trapping level, generate difference kind of defect and clusters.

Doping atom diffuse to substrate bulk by randomly and no direction. Atom will diffuse to closely area and where defect and vacancy has in mechanism. However, doping atom will replace host lattice position or interstitial. However, atom diffusion of results follow Fick's law by [14]

$$
\mathrm{J}=-\mathrm{D}(\mathrm{d} \varphi / \mathrm{d} x)
$$

Where: $\mathrm{J}$ is diffusion flux, $\mathrm{D}$ is diffusion coefficient, $\varphi$ is concentration and $x$ is position (dimension).

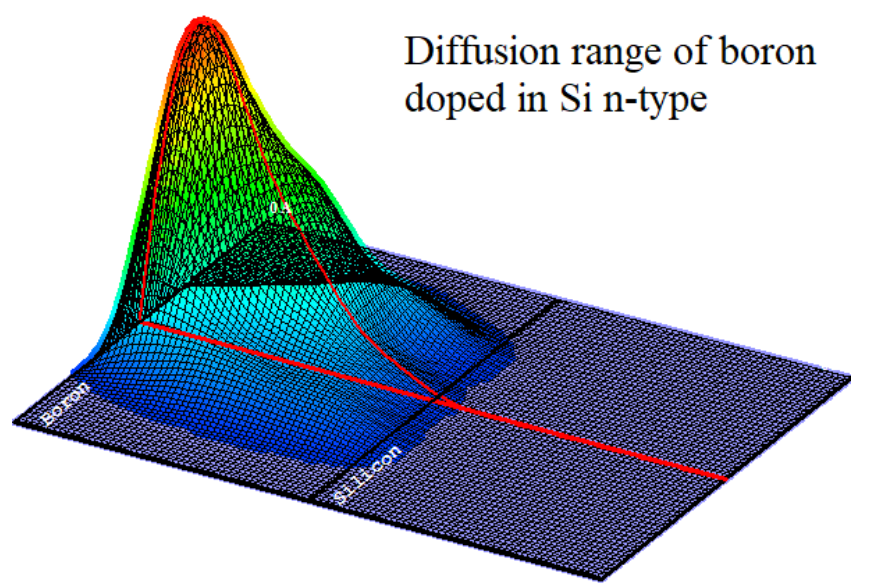

Fig. 4 Diffusion range simulation results

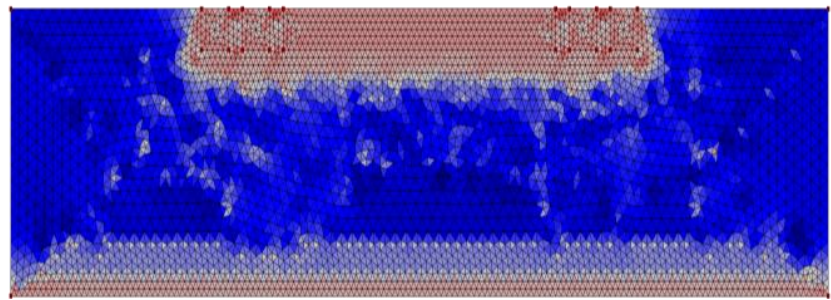

Fig. 5 Doping atom diffused in silicon substrate

Fig. 5 shows doping atom in silicon mechanism, the atom will diffuse by non-direction to substrate. By the corner of guard ring has doping atom accumulate, btw atom diffuse to area lower and around diffusion zone. This will impact for substrate bulk such generate defect and change properties of device.

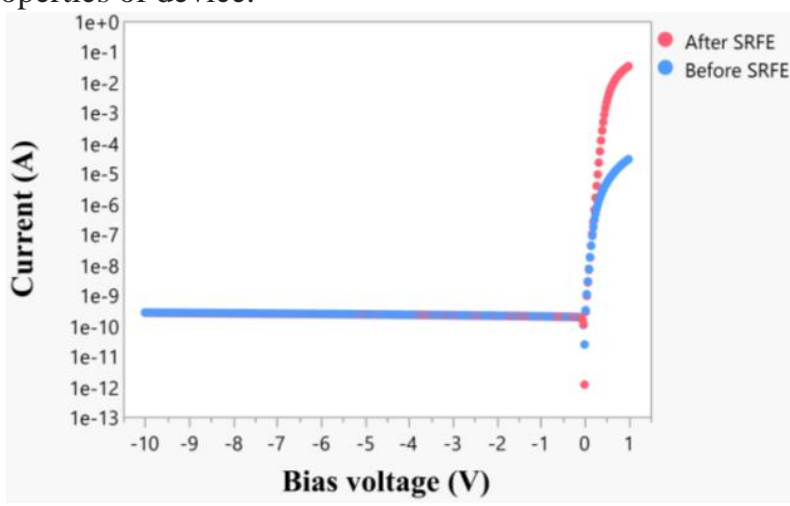

Fig. 6 Current-voltage before and after SRFE process (red-before SRFE, blue-after SRFE)

The electrical properties of device before and after SRFE process shown in Fig. 6, 7. Results show reverse bias -10 to $0 \mathrm{~V}$ by step $0.02 \mathrm{~V}$. SRFE may change or help to reduce defect in P-N diode. The leakage current before expose radiation show around $2.65 \times 10^{-10} \mathrm{~A}$ at $-10 \mathrm{~V}$. Radiation expose to device by 40 seconds (multi-time exposed), after exposed $40 \mathrm{sec}$ leakage current reduced around $2.6 \times 10^{-10} \mathrm{~A}$. From the results we can explain that SRFE may help to improve performance of P-N diode although the number of leakage current not significant but it good start for using SRFE process because many research always publish influence from radiation on semiconductor device such as neutron damage to device and photon increase leakage current. [15]

Published By:

Blue Eyes Intelligence Engineering DOI: 10.35940/ijeat.D7683.049420

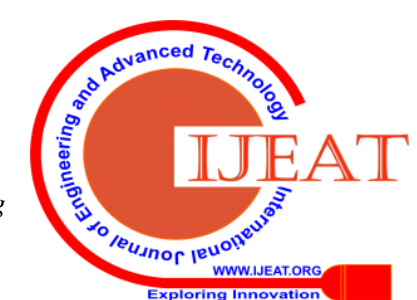




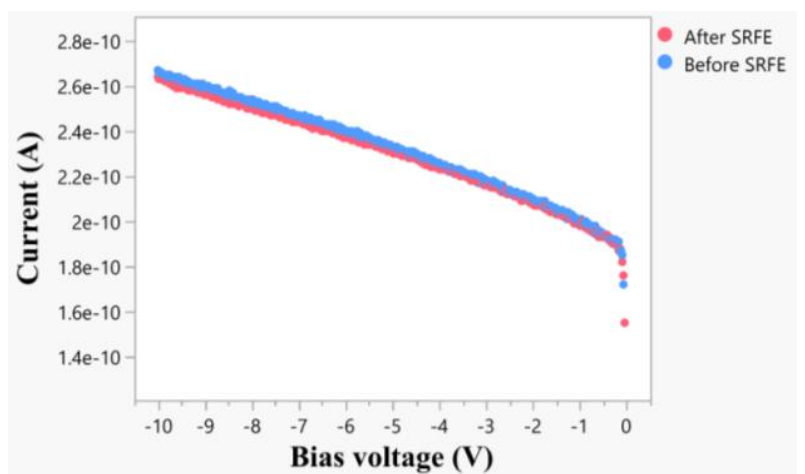

Fig. 7 Reverse current compare between before and after SRFE process

From Fig. 7 results, SRFE process may get optimize energy and time for exposed to treatment on device. From simulation results doping process may generate defects in device mechanism and SRFE help to reduced defects.

\section{CONCLUSIONS}

This paper focus the effect of SRFE process on leakage current properties of $\mathrm{P}-\mathrm{N}$ diode. SRFE process take many shots to expose radiation on device by overall time $40 \mathrm{sec}$. Simulation results show diffusion atom deep to substrate mechanism by random and no direction. Atom may create cluster and defects but after SRFE process with $40 \mathrm{sec}$ the leakage current shown a bit reduce. This mean, SRFE process reduced leakage current of device. Although, exposed time $40 \mathrm{sec}$ can reduce leakage current not much but this is good results for using radiation for treatment semiconductor device. However, we will study more impact and try to expose wide range of radiation to present in future.

\section{ACKNOWLEDGMENT}

The authors would like to thank Suan Sunandha Rajabhat University (SSRU) and King Mongkut's University of Technology North Bangkok (KMTNB) for providing the $\mathrm{X}$-ray exposure equipment for this experiment, Thai Microelectronics Center (TMEC) for fabrication P-N junction diode, National Electronics and Computer Technology Center, Thailand and Thailand Graduate Institute of Science and Technology (TGIST).

\section{REFERENCES}

1. J. A. Alamo, "Measurement of steady-state-minority-carrier transport parameters in heavily doped n-type silicon," IEEE T Electron Dev, vol. 34 Jul. 1987, pp. 1580-1589.

2. A. Sahu, L. K. Bramhane, and J. Singh, "Symmetric lateral doping-free BJT: A novel design for mixed signal application," IEEE T Electron Dev, vol. 63 May. 2016, pp. 2684-2690.

3. W. Kuzmicz, "The future of CMOS: more Moore or the next big thing," 2017 Mixed design of integrated circuits and systems, vol. 1 Jun. 2017, pp. 17097624.

4. J. Hornberge, A. B. Lostetter, and K. J. Olejniczak, "Silicon-carbide (SiC) semicounductor power electronics for extreme high-temperature environment," 2004 IEEE Aerospace conference proceeding, vol. 1 Mar. 2004, pp. 8255610.

5. A. Barnett, C. Honsberg, and D. Kirkpatrick, "50\% efficient solar cell architectures and designs," 2006 IEEE $4^{\text {th }}$ world conference on photovoltaic energy conference, vol. 1 May. 2006, pp. 9241110.

6. I Kandarakis, D. Cavouras, and D. Nikolipoulos, "A theoretical model evaluating the angular distribution of luminescence emission in X-ray scintillating screens,” Appl. Radiat. Isot., vol. 64 Apr. 2006, pp. 508-519.

7. P. K. Guha, S. Z. Ali, and C. C. C. Lee, "Novel design and characterization of SOI CMOS micro-hotplates for high temperature gas sensors," Sensor and Actuator B, vol. 127 Oct. 2007, pp. 260-266.

8. P. M. Johns, and J. C. Nino, "Room temperature detectors for nuclear security,” J Appl Phys, vol. 126 Jun. 2019, pp. 040902-1-20.

9. H. W. Kraner, "Radiation damage in semiconductor detectors," IEEE Trans. Nucl. Sci., vol. 29 Jun. 1982, pp. 1087-1100.

10. A. Ruzin, G. Casse, and M. Glaser, "Comparison of radiation damage in silicon induced by proton and neutron irradiation," IEEE Trans. Nucl. Sci., vol. 46 Oct. 1999, pp. 1310-1313.

11. A. Raulo, M. Sowinska, and G. Hennard, "Characteristics of X-and Gamma-ray CdTe radiation detectors," 2011 IEEE Nuclear Science symposium conference record, vol. 1 Oct. 2011, pp. 12557447.

12. S. Niemcharoen, S. Ueamanapong, and I. Srithanachai, "Removing the transients electron trapping in P-N junction diode by using soft X-ray annealing method," J Nanoelectron, vol. 7 Jun. 2014, pp. 72-80.

13. I. Srithanachai, S. Ueamanapong, and A. Poyai, "An experimental investigation of P-N diode electrical characteristics by soft X-ray annealing method," Opt Laser Technol, vol. 44 Sep. 2011, pp. 635-639.

14. J. R. Lowney, and R. D. Larrabee, "The use of Fick's law in modeling diffusion processes," IEEE T Electron Dev, vol. 27 Sep. 1980, pp. 1795-1798.

15. K. Zhang, D. Peng, and W. C. Chong, "Investigation of photon-generated leakage current for high-performance active matrix micro-LED displays," IEEE T Electron Dev, vol. 63 Dec. 2016, pp. 4832-4838.

\section{AUTHORS PROFILE}

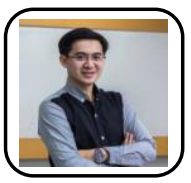

Narong Sangwaranatee received the B.S. degree in physics in Thepsatri Rajabhat University in 2003, and the M.S. and Ph.D. degree in applied physics both from the King Mongkut's Institute of Technology Ladkrabang, Bangkok, Thailand, in 2006 and 2009, respectively. He is currently an Associate Professor with the Department of applied physics, Faculty of Science and Technology, Suan Sunandha Rajabhat University, Bangkok and his research interests solid state electronics and solid state lighting.

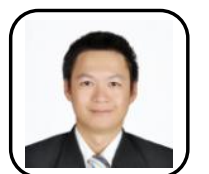

Itsara Srithanachai He received the B.S. degree in applied physics in 2006, the M.Eng and Ph.D. degree in electronics engineering in 2009 and 2013 both from the King Mongkut's Institute of Technology Ladkrabang, Ladkrabang, Thailand. His master degree research was in the field of photodetector process and Ph.D study on radiation effect on semiconductor device. He has 8 years of experience in semiconductor fabrication process.

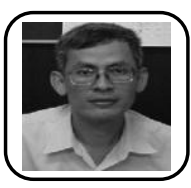

Surasak Niemcharoen received the B.S. degree in physics from Chiang Mai University, Chiang Mai, Thailand, in 1985, and the M.Eng. and D.Eng. degrees in electrical engineering both from the King Mongkut's Institute of Technology Ladkrabang, Bangkok, Thailand, in 1992 and 2004, respectively. He is currently an Assistant Professor with the Department of Electronics and Electronics Research Center, Faculty of Engineering, King Mongkut's Institute of Technology Ladkrabang and his research interests include metal-semiconductor (MS) devices and metal-semiconductor-metal (MSM) photodetectors based on silicon technology.

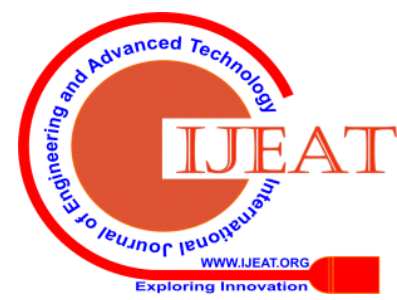

